\title{
Kinesiologisches Taping in der Osteopathie
}

\author{
Sascha Seifert
}

Wer kennt sie nicht, die bunten Klebestreifen, die seit vielen Jahren immer wieder auf Rückenpartien, Fußgelenken, Schultern oder Nacken von Sportlern angebracht sind? Was hat es damit auf sich und wie lässt sich diese Art des Tapens in die osteopathische Behandlungspraxis integrieren?

Unter kinesiologischem Taping versteht man eine Therapie, bei der elastische, auf der Haut haftende Streifen mehr oder weniger gedehnt auf die Haut geklebt werden. Anders als ein starres Tape, das in der Sportmedizin zur Ruhigstellung und Stabilisierung eingesetzt wird, bleibt ein kinesiologisches Tape elastisch und flexibel. Und das muss auch so sein. Denn das kinesiologische Tape entfaltet seine Wirkung erst bei Bewegung.

\section{Entwicklung des Kinesio-Tapings}

Die Methode des kinesiologischen Tapings kommt ursprünglich aus Japan. Sie wurde in den 1970er-Jahren von dem japanischen Arzt und Chiropraktiker Dr. Kenzo Kase entwickelt. Er suchte nach einem Weg, Schmerzen auf natürliche Weise zu lindern. Seine ersten Patienten waren lange Zeit Sumo-Ringer. Den Weg nach Europa fand das Tape durch den Profifußball. Der Entwicklung des Kinesio-Tapings lagen folgende Überlegungen zugrunde: Sportverletzungen sollten nicht mehr durch Ruhigstellung der Gelenke behandelt werden, sondern durch eine Methode, die bewusst die Mobilisation des Stützund Bewegungsapparats in den Vordergrund stellt, zudem sollten die Nachhaltigkeit der manuellen Behandlung erhöht und gleichzeitig die immer wieder auftretenden negativen Auswirkungen durch die konventionelle chiropraktische Behandlung möglichst gering gehalten werden.

Die International Taping Association (ITA) hat die Grundlagenarbeit von Kase weiterentwickelt und sie mit den Erkenntnissen der Faszienforschung und Osteopathie erweitert.

Ziel des kinesiologischen Tapings ist es, durch die verschiedenen Anlagetechniken, die körpereigenen Selbstheilungskräfte zu unterstützen. Damit ist über die Wirkungsweise und den Sinn des Tapes eigentlich schon alles gesagt - frei nach A.T. Still: „Find it, tape it and leave it alone.“ In diesem Sinn kann das Tapen eine sinnvolle Ergänzung sein, die den Körper nach der Behandlung durch den Osteopathen positiv unterstützt und die Selbstregulation verbessert. Besonders im viszeralen Bereich gibt es im Rahmen des vizeralen Tapings zahlreiche Erfolg versprechende Behandlungsansätze.

\section{Basiskonzepte}

Es kann davon ausgegangen werden, dass das auf die Haut aufgeklebte Tape die darunterliegenden Gewebeschichten bewegt, Schmerzsensoren beeinflusst, Muskelkontraktionen, Lymphfluss und Gelenkfunktion stimuliert. Zudem werden Nervenzellen angeregt und die Durchblutung in dem betreffenden Bereich gefördert. Es gibt im Kinesio-Taping 4 Basiskonzepte [6], welche die Wirkmechanismen zu erklären versuchen, obwohl die genaue Wirkungsweise nach wissenschaftlichen Gesichtspunkten bis heute noch nicht genau geklärt ist. Diese Konzepte stehen in Relation und Wechselwirkung zueinander und werden durch das Tape beeinflusst.

- Faszienkonzept

- Schmerzkonzept
- Mikrozirkulationskonzept (hier nicht weiter aufgeführt)

- Hautkonzept

\section{Faszienkonzept}

Beim Taping werden in der Regel die oberflächlichen Schichten der Fascia superficialis erreicht, da diese direkt mit der Haut in Verbindung stehen. Eine neue Studie von Tu, Woledge und Morrissey [8] belegt, dass sich die Beweglichkeit des subkutanen Gewebes der Fascia thoracolumbalis durch Kinesiotape verbessert.

$\mathrm{Zu}$ den medialen und tiefen Schichten besteht nur ein indirekter Zugang. Wie Tesarz [7] beschreibt, vermitteln die Vater-Pacini-Körperchen innerhalb der Fascia superficialis afferente Informationen an das Zentralnervensystem (ZNS) und haben damit einen bedeutenden Einfluss auf den neuromuskulären Reflexmechanismus und die Körperwahrnehmung. Tu, Woledge und Morrisse zeigen auf, dass sich durch kinesiologische Tapes die Beweglichkeit des subkutanen Gewebes verbessert [8].

Faszien haben die Eigenschaft, auf akute Verletzungen oder chronische repetitive Mirkrotraumatisierungen zu reagieren. Besonders in der oberflächlichen Faszienschicht ist nach Bove [1] durch den relativ großen Raum die Möglichkeit gegeben, dass sich Exsudat sammelt. Dieses wird normalerweise resorbiert. Durch spezielle Tapeanlagen (Webcuts, Lymphanlagen und Muskeltechniken mit Convolutions, d.h. einer Wellenbildung des Tapes mit der Haut) kann direkt Einfluss auf diese Prozesse genommen werden, weil der Abtransport des Exsudats in die Lymphgefäße verbessert wird.

Indem es die oberste Hautschicht anhebt, wird der Blut- und Lymphfluss in der darunterliegende Gewebeschicht an- 


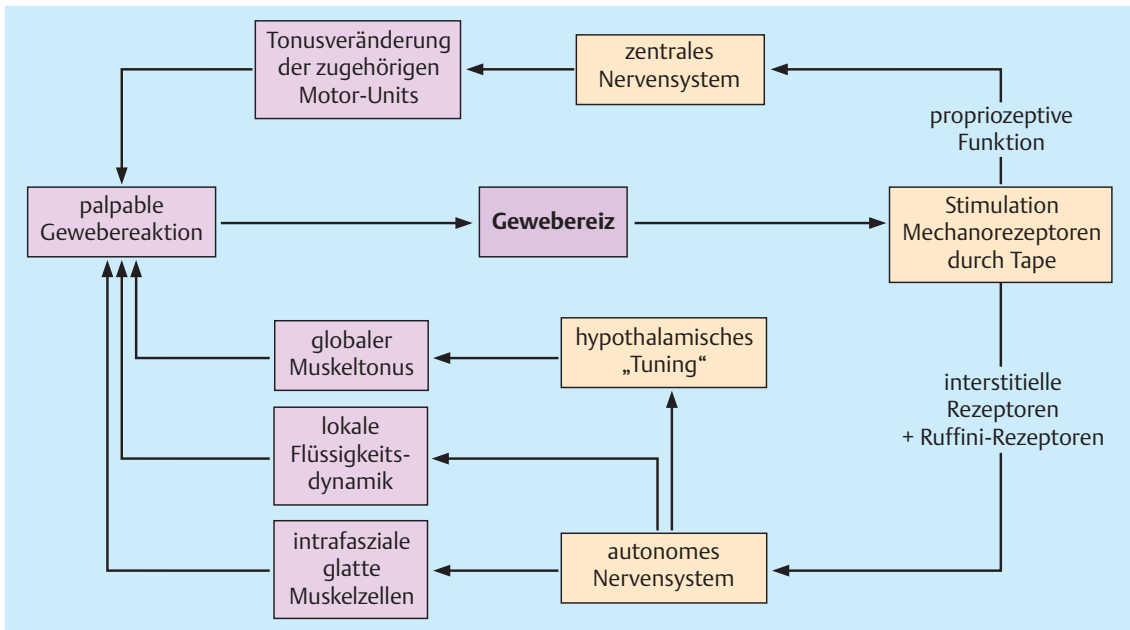

Abb. 1 Wirkung des Tapes (mechanorezeptiver Stimulus). Quelle: [6]

geregt. Nährstoffe und Abwehrkörper können besser angeliefert, Abfallprodukte und Gewebswasser schneller abtransportiert werden.

\section{Schmerzkonzept}

Es wird davon ausgegangen, dass das Tape einen neuen, permanenten Reiz auf die Haut ausübt, der sich mit schon länger bestehenden Schmerzreizen überlagert und diese dadurch positiv beeinflusst.

\section{Hemmung der Nozizeption durch Stimulation der Propriozeptoren}

Die Schmerzwahrnehmung der nozizeptiven Afferenzen (C-Fasern, Aס-Fasern) wird auf das Wide-Dynamic-Range-Neuron (WDR-Neuron) im Hinterhorn des Rückenmarks projiziert. Neben diesen tief liegenden somatischen Afferenzen („deep somatic afferences“) werden auch die propriozeptiven Reize aus der Haut, den Muskeln, den Gelenken und den Viszera dort hingeleitet. Die zentrale Schmerzwahrnehmung beruht auf dem Überlaufprinzip des WDR-Neurons. Dieses hat eine Konvergenzfunktion, weshalb eine exakt lokalisierbare Differenzierung der Afferenzen nicht möglich ist. So wird der Schmerz unabhängig von der Quelle, vom Thalamus oder der Großhirnrinde irgendwo im Segment lokalisiert. Dies kann als „zentrale Wahrnehmungstäuschung“ bezeichnet werden. Besonders ausgeprägt sind diese Schmerzprojektionen bei myofaszialen Störungen und verstärkter zentraler Sensibilisierung. Durch eine Stimulation oder Entlastung der propriozeptiven Afferenz, z.B. durch ein Tape, wird wahrscheinlich die Information verändert und es kann zur Schmerzreduktion kommen ( $\triangleright$ Abb. 1).

\section{Einfluss des Tapes auf die WDR-Neuronen}

Diese multirezeptiven Neuronen sind wahrscheinlich sehr gut durch das Tape beeinflussbar, weil sie auf leichte Reize wie Berührung oder Haarbewegungen reagieren. Häufig werden diese Neuronen durch eine schmerzhafte Reizung in der Peripherie (Haut, Muskeln, Gelenke, Gefäße, Meningen) und des Interstitiums aktiviert - so kann das Phänomen der Konvergenz erklärt werden.

\section{Hautkonzept}

Mit den Tapes wird zu 100\% auf der Haut gearbeitet, deshalb ist die Funktion dieses Organs für das Taping von enormer Bedeutung.

Beim durchschnittlichen Erwachsenen beträgt die Größe der Haut ca. 1,2-2,3 m². Das Gewicht der Haut entspricht etwa 16\% des Körpergewichts eines Menschen, womit sie das größte und schwerste Organ unseres Körpers ist. Sie dient als Grenze (externes/internes Milieu), Schutz (chemisch, mechanisch, thermisch) sowie als Entgiftungsorgan. Außerdem ist sie ein hervorragendes Wahrnehmungsorgan. Die Gesamtlänge aller Nervenbahnen in der Haut wird auf $80 \mathrm{~km}$ geschätzt. Die verschiedenen Messfühler haben die Aufgabe, physische Reize der Umwelt in die Sprache des Körpers zu übersetzen. So sind sie in der Lage, Drücke ab einem Gewicht von 0,31 mg wahrzunehmen. Durch die Haftung des Tapes auf der Haut und die dadurch ausgelöste mechanische Verschiebung reizt das Tape bei ausgeführten Körperbewegungen die Rezeptoren in der Haut. Diese Informationen laufen wie der Schmerzreiz aus dem betroffenen Gewebe über Nerven in das gleiche Tor (Hinterhorn) ins Rückenmark. Diese sind stärker und schneller als die Schmerzreize und hemmen damit die Schmerzweiterleitung. Es kommt zu einer Schmerzlinderung. 


\section{Wirkung des Tapes über Dermatome}

Grundlegende Erkenntnisse über Segmente der Wirbelsäule bzw. Dermatome der Haut wurden schon von Head, McKenzie, Hansen, Dittmar und von Staa gesammelt. Hierbei handelt es sich jedoch ausschließlich um klinische Untersuchungen und Beobachtungen, wie Spinalnerven bestimmte Hautbezirke versorgen (Dermatome) und umgekehrt bestimmte Hautbezirke zugeordnete Spinalnerven (bzw. Segmente) versorgen. Laut Teirich-Leube konnten wissenschaftliche Erklärungen nicht erfolgen, da bei anatomisch-präparatorischen Untersuchungen viszerosensible Fasern und efferente vegetative Nervenfasern nicht voneinander $\mathrm{zu}$ unterscheiden sind. Erschwerend kommt hinzu, dass sich beide Nervensysteme in ihrem Verlauf mehrfach kreuzen. Dies hat schon der Franzose Georgia Knap (1866-1946) bei zahlreichen Experimenten herausgefunden. Auf der Suche nach ewiger Jugend stieß er auf 18 Punkte, die bei Dysfunktionen der Viszera besonders schmerzhaft sind und die Homöostase des Körpers stören. Er nannte sie „nervale Kreuzung“. Auch andere angesehene Osteopathen haben die Verbindungen von Hautveränderungen in spezifische Zonen mit viszeralen Dysfunktionen dokumentiert. So erstellte Dr. Henri Jarricot bereits 1975 eine topografische Karte der Haut, auf der präzise Stellen beschrieben sind, die in Verbindung mit den viszeralen Organen stehen.

Der Osteopath Frank Chapman war überzeugt, dass die knöchernen osteopathischen Dysfunktionen nicht die gesamte Pathologie erklären können. Nach jahrelangen Untersuchungen und Experimenten fand er 48 Punkte auf der Haut, die er einzelnen Organen zuordnete. Chapman beschrieb Gewebsveränderungen, welche tastbar, aber bildlich nicht darstellbar sind. Somit nannte er die Punkte „neurolymphatische Reflexe“. Er war überzeugt, dass sich eine Stase der Lymphe in den viszeralen Organen als Schmerz an den distalen Spinalnervenendigungen darstellt (viszerokutane Reflexe).

Auch andere Therapien haben diese Relationen gefunden und nutzen sie. Unter anderem finden sie Anwendung in der Triggerpunkttherapie oder in der Periostmassage. Hier entdeckte Prof. Vogler 1928 den Zusammenhang zwischen Periostschmerz und spezifischen funktionellen Problemen in den Viszera.

\section{Wirkungen des Tapes über den viszerokutanen und kutiviszeralen Reflex}

Der viszerokutane Reflex ist für die diagnostische Untersuchung sehr wichtig. Hierbei erfolgt die Reizausbreitung vom kranken Organ zur Peripherie.

Der vom Organ ausgehende Nervenreiz verläuft über die vegetativen Nervenbahnen durch den Grenzstrang. Der Reiz läuft über den Ramus communicans griseus des zugehörigen Segments durch die Hinterhornsäule hindurch in die Seitenhornsäule. Von der Seitenhornsäule erfolgt die Reizübertragung zur Intermediärzone. Durch das Vorderhorn verlässt das Reizpotenzial das Rückenmark, ohne im Rückenmark selbst auf- oder abzusteigen. Über den Ramus communicans albus wird das Grenzstrangganglion als Schaltneuron erreicht. Vom sympathischen Grenzstrangganglion gelangt der Reiz über den Ramus communicans griseus zum subkutanen Bindegewebe. Somit kommt es im Bindegewebe und in der Kutis zu Veränderungen, die man ertasten kann. Jarricot und Chapman haben zahlreiche Regionen beschrieben, die zu diesem Prinzip passen.

Bei dem kutiviszeralen Reflex erfolgt die Reizausbreitung von der Peripherie zum Organ. Dies ist ein wichtiges Kriterium für die Therapie. Die Behandlung und Einflussnahme auf viszerale Dysfunktionen findet sich in bekannten Therapien wie Bindegewebs-, Fußreflexzonen- und Periostmassagen wieder.

Durch einen Reiz der Kutis beginnt der kutiviszerale Reflexbogen. Der Reiz wird über die sensiblen Bahnen, über das Spinalganglion zur Radix posterior und weiter zur Seitenhornsäule geleitet. Von hier gelangt der Reiz über den Ramus communicans albus und das Grenzstrangganglion zum Organ.

\section{Viszerales Taping}

Viszerales Taping wurde 2013 von der ITA entwickelt und ist eine hervorragende Ergänzung zu den parietalen Tapeanlagen. Nach einer osteopathischen Behandlung kann eine Tapeanlage sinnvoll sein, um die physiologischen Prozesse positiv zu unterstützen. Diese Anlagen werden 5-7 Tage an der Stelle getragen, an der sich der Osteopath eine Wirkungsverstärkung

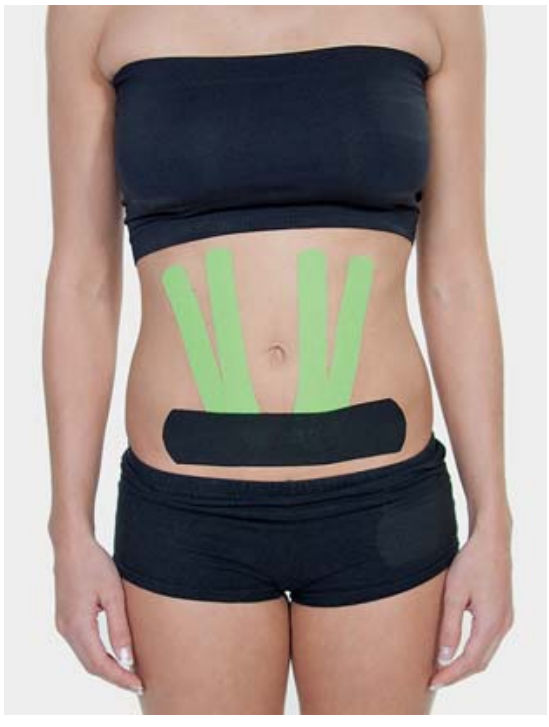

- Abb. 2 Anlage Ptosis visceralis. Quelle: [6]

der Behandlung wünscht. Die Effekte des Tapes z.B. bei der Anlage Ptosis visceralis können vorher mit einem Inhibitionstest überprüft werden. Durch den konsequenten Einsatz von Inhibitions- und Faszientest (Screeningtests) kann die Wirkung der Tapeanlage vom Osteopathen deutlich verbessert werden. Durch diese Tests werden Ferneinflüsse auf ein lokales Problem überprüft, somit können auch Tapeanlagen indirekt wirken. Zudem lässt sich mithilfe des Inhibitionstests (Screeningtest) häufig nachweisen, dass Bewegungseinschränkungen der Extremitäten durch viszerale Dysfunktionen bedingt sind.

\section{Viszerale Anlage bei Ptose}

Diese Anlage hat die Funktion, eine Ptose nach superior zu entlasten. $\mathrm{Zu}$ beachten sind die Hautfalten unter dem Tape, damit es nicht zur Verletzung der Haut kommt. Zur Anwendung kommen 2 Y-Tapes und 1 I-Tape von etwa $20 \mathrm{~cm}$ Länge ( $\bullet$ Abb. 2).

- Indikationen: Ptosis visceralis, Dysfunktion der Blase, Dysfunktion des Uterus

- Techniken: Faszientechnik mit 25-75prozentigem Zug, funktionelle Korrektur mit 50-75-prozentigem Zug

\section{Viszerales Gallenblasen-Tape}

Bei der Behandlung von Kongestionen der Gallenblase kann eine Tapeanlage ( $\triangleright$ Abb.3) unterhalb des rechten Rippenbogens für mehrere Tage deutlich die Spannung der faszialen Strukturen in der 


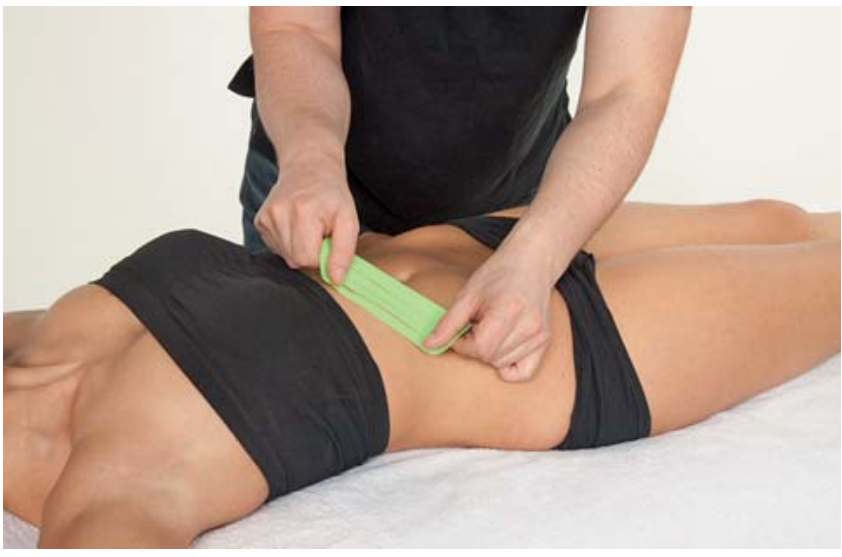

- Abb.3 Anlage Gallenblase. Quelle: [6]

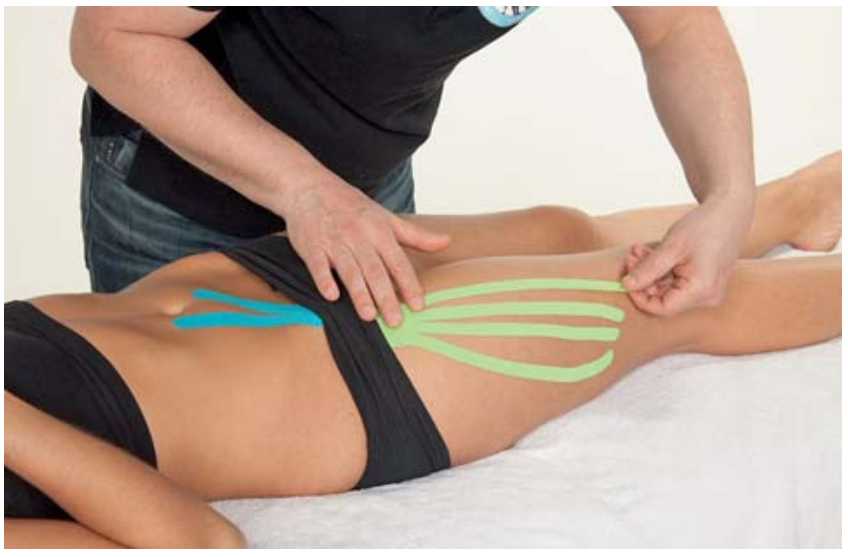

- Abb. 4 Anlage Regio ingunialis. Quelle: [6]
Region positiv beeinflussen. Aufgebracht wird 1 I-Tape.

- Indikationen: Kongestion der Gallenblase, Mikrolithiasis, Dysfunktion des Ductus choledochus

- Techniken: Muskeltechnik mit 0-prozentigem Zug

\section{Tape in der Regio inguinalis}

In diesem Bereich kommt es häufig zu Kompressionssyndromen der nervalen und vaskulären Strukturen. Durch eine Tapeanlage ( Abb.4) kann mithilfe der Faszientechnik eine dauerhafte Entlastung geschaffen werden. Eingesetzt werden 2 Faszientapes mit Y-Schnitt.

- Indikationen: Dysfunktionen des N. cutaneus femoris lateralis, N. femoralis, der A. und V. femoralis, Ramus femoralis des N. genitofemoralis, V. saphena accessoria lateralis, V. saphena magna, A. und V. pudenda externa, Hiatus saphenus sowie bei Dysfunktionen im Bereich des M. iliopsoas und M. sartorius

- Techniken: Faszientechnik mit 25-75prozentigem Zug

\section{Wirkungen}

Wie Krajczy, Bogacz, Lunieski et al. [3] in einer Studie zur Atonie des Darmes nach Choleozystektomie (direkt postoperativ) nachweisen konnten, haben Tapes einen positiven Einfluss auf die Defäkation und auf viszerale Schmerzen.

Für die Beeinflussung der Viszera über Tapes eignet sich der Behandlungsansatz von Finet und Willame [2]. Deren in den 1980er-Jahren durchgeführte Studien zur Bewegung von Organen in Abhängigkeit von der Atembewegung des Diaphragma abdominale zeigten, dass die Organbe- weglichkeit durch Intervention am ventralen Peritoneum parietale beeinflusst werden kann. Sie beschrieben ihre Behandlung als faszial, weil sie das Peritoneum als Faszie betrachtet haben. Viele Tapeanlagen im viszeralen Bereich werden auf der Haut im Bereich des Peritoneum parietale anterius mit einer sog. Faszien-Technik durchgeführt.

\section{Literatur}

1 Bove GM, Chapelle SL. Visceral mobilization can lyse and prevent peritoneal adhesions in a rat model. Journal of bodywork and movement therapies. 2012; 16(1): 76-82

2 Finet G, Williame C. Treating Visceral Dysfunction: An Osteopathic Approach to Understanding and Treating the Abdominal Organs. Portland: Stillness Press; 2000

3 Krajczy M, Bogacz K, Luniewski J et al. The influence of Kinesio Taping on the effects of physiotherapy in patients after laparoscopic cholecystectomy. ScientificWorldjournal. 2012 (2012) Article ID: 948282, doi:10.1100/ 2012/948282

4 Morris D, Jones D, Ryan H et al. The clinical effects of Kinesio ${ }^{\circledR}$ Tex taping: A systematic review. Physiother Theory Pract. 2013; 29(4): 259-70

5 Schleip R, Jäger H. Faszien und ihre Bedeutung für die Interozeption. Osteopathische Medizin 2014; 15(3): 25-30

6 Seifert S. Kinesiologisches Taping in der Osteopathie und Manuellen Therapie. Stuttgart: Haug; 2015

7 Tesarz J. Die Fascia thoracolumbalis als potenzielle Ursache für Rückenschmerzen: anatomische Grundlagen und klinische Aspekte. Osteopathische Medizin 2010; 11(1): 28-34

8 Tu JS, Woledge R, Morrissey D. Measurement of the Effects of "Kinesio-Taping" in Vivo Thoracolumbar Fascia Movement Using
Ultrasound: Method Development and Observational StudyMicrosoft. Available at: http://www.fasciacongress.org/2015/ Abstracts/112_Tu.pdf

\section{Online}

http://dx.doi.org/10.1055/s-0035-1557849

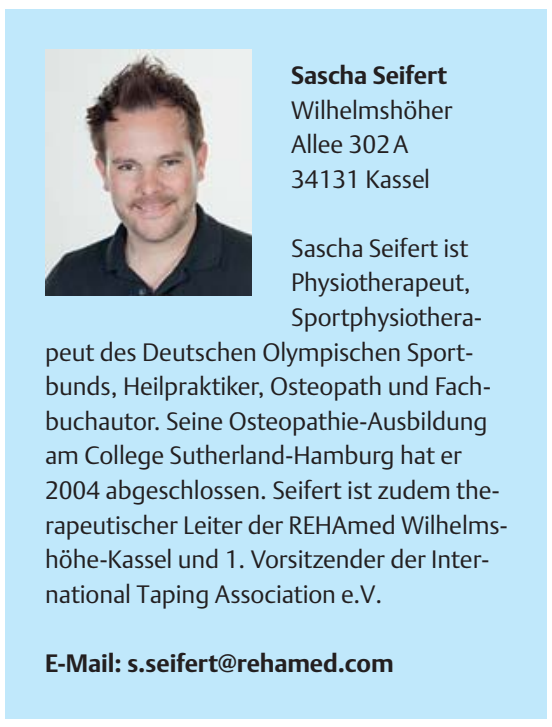

